\title{
Resistencia a antibióticos no betalactámicos de aislamientos invasores de Streptococcus pneumoniae en niños latinoamericanos. SIREVA II, 2000-2005
}

\author{
Clara Inés Agudelo, ${ }^{1}$ Elizabeth Castañeda, ${ }^{1}$ Alejandra Corso, ${ }^{2}$ Mabel Regueira, ${ }^{2}$ \\ Maria Cristina de Cunto Brandileone, ${ }^{3}$ Angela Pires Brandão, ${ }^{4}$ Aurora Maldonado, ${ }^{5}$ \\ Juan Carlos Hormazabal, ${ }^{5}$ Isis Tamargo, ${ }^{6}$ Gabriela Echániz-Aviles, ${ }^{7}$ Araceli Soto, ${ }^{7}$ \\ Mónica Guadalupe Viveros, ${ }^{8}$ Irma Hernández, ${ }^{8}$ Gustavo Chamorro, ${ }^{9}$ Natalie Weiler, ${ }^{9}$ \\ Jacqueline Sánchez, ${ }^{10}$ Jesús M. Feris, ${ }^{10}$ Teresa Camou, ${ }^{11}$ Gabriela García, ${ }^{11}$ \\ Enza Spadola, ${ }^{12}$ Daisy Payares, ${ }^{12}$ Jean-Marc Gabastou, ${ }^{13}$ José Luis Di Fabio ${ }^{13}$ \\ y el Grupo SIREVA I/ ${ }^{14}$
}

Forma de citar

Agudelo CI, Castañeda E, Corso A, Regueira M, Brandileone MCC, Brandão AP, et al. Resistencia a antibióticos no betalactámicos de aislamientos invasores de Streptococcus pneumoniae en niños latinoamericanos. SIREVA II, 2000-2005. Rev Panam Salud Publica. 2009;25(4):305-13.

RESUMEN Objetivo. Determinar la evolución de la resistencia a la eritromicina, el cloranfenicol, el trimetoprim-sulfametozaxol (SXT) y la vancomicina de aislamientos invasores de Streptococcus pneumoniae obtenidos de niños de 10 países de América Latina y del Caribe en seis años de vigilancia.

Métodos. Se analizaron 8993 aislamientos de S. pneumoniae recuperados entre 2000 y 2005 de niños menores de 6 años con infecciones invasoras, procedentes de Argentina, Brasil, Chile, Colombia, Cuba, México, Paraguay, República Dominicana, Uruguay y Venezuela. La sensibilidad a los antibióticos se determinó mediante los métodos establecidos y estandarizados en el proyecto SIREVA. La resistencia a múltiples antibióticos se definió como la resistencia a tres o más familias de antibióticos, de los no betalactámicos analizados en este estudio o de los betalactámicos evaluados en un estudio previo en el que 37,8\% de estos aislamientos presentaron sensibilidad disminuida a la penicilina.

Resultados. Se encontró algún grado de resistencia al SXT y la eritromicina $(56,4 \%$ y $15,4 \%$ de los aislamientos estudiados, respectivamente) y 4,6\% presentó alta resistencia al cloranfenicol. Todos los aislamientos fueron sensibles a la vancomicina. Se observó la mayor frecuencia de resistencia al SXT en los aislamientos de neumonía y a la eritromicina en los casos de sepsis $(61,6 \%$ y 25,5\%, respectivamente; $\mathrm{P}<0,01)$. La mayor frecuencia de resistencia al SXT se observó en Brasil (71,9\%) y a la eritromicina en México (38,2\%) y Venezuela (32,9\%). Los serotipos $14,6 B, 19 F$ y $23 F$ fueron los que más frecuentemente se asociaron con la resistencia a los antibióticos estudiados.

La afiliación de los autores y los miembros del grupo SIREVA II, todos coautores del presente trabajo, se presentan al final del artículo. La corres- pondencia se debe dirigir a Clara Inés Agudelo, Calle 115 No. 47A-14, apartamento 307, Barrio La
Alhambra, Bogotá, D.C., Colombia. Correo electrónico: cia1949@gmail.com 
Conclusiones. Se observó una elevada y creciente frecuencia de aislamientos resistentes al SXT y la eritromicina, y una disminución en la proporción de aislamientos resistentes al cloranfenicol. Estas tendencias mostraron diferencias entre los países estudiados.

Palabras clave
Agentes antibacterianos, Streptococcus pneumoniae, resistencia beta-lactámica, niños, América Latina.
Streptococcus pneumoniae ocasiona enfermedades invasoras como la meningitis, la neumonía y la sepsis. Este patógeno constituye un problema prioritario de salud pública debido a las elevadas tasas de morbilidad y mortalidad que provoca, especialmente en la población infantil (1), y al incremento creciente de su resistencia a los antibióticos (2-6).

La Organización Mundial de la Salud (OMS) propuso en 2001 una estrategia mundial para contener la resistencia a los antimicrobianos (7), en la que se subraya la importancia de que cada país establezca la vigilancia de la resistencia a los antibióticos de los microorganismos causantes de enfermedades de importancia para la salud pública, entre los que se encuentra $S$. pneumoniae. Esta vigilancia tendría como finalidad obtener información sobre la magnitud y las tendencias del problema y, posteriormente, realizar el seguimiento a las intervenciones que se realicen en cada país, en particular la aplicación de medidas para el uso racional de los antibióticos. Además, los datos nacionales también son importantes para establecer los patrones específicos de resistencia, que varían considerablemente de un país a otro y a lo largo del tiempo (7).

La Organización Panamericana de la Salud (OPS) creó en 1994 el programa SIREVA (Sistema Regional de Vacunas) para la vigilancia de las infecciones invasoras de S. pneumoniae en niños latino- americanos menores de 6 años $(3,8)$. A partir de 2004, este programa se denomina Sistema de Redes de Vigilancia de los Agentes Bacterianos Responsables de Neumonía y Meningitis, SIREVA II (9). El objetivo de este programa es conocer la distribución de los diversos serotipos y registrar la evolución de la resistencia a los antibióticos de elección para el tratamiento de esas infecciones, a saber betalactámicos, macrólidos, cloranfenicol, trimetoprim-sulfametozaxol (SXT) y glicopéptidos (3-5, 9-10).

En un estudio reciente se analizó la sensibilidad a los antibióticos betalactámicos de 8993 cepas de $S$. pneumoniae aisladas en menores de 6 años con infecciones invasoras en 10 países de América Latina y el Caribe entre los años 2000 y 2005 (11). Se estableció que $62,2 \%$ de los aislamientos era sensible a la penicilina $(22,1 \%$ presentó resistencia intermedia y $15,7 \%$ resistencia alta), mientras que $85,6 \%$ de los aislamientos estudiados resultaron sensibles a la ceftriaxona $(11,4 \%$ presentaron resistencia intermedia y $3,1 \%$ resistencia alta). En el presente trabajo se analiza la evolución de la resistencia a antibióticos no betalactámicos (eritromicina, cloranfenicol, SXT y vancomicina) de esos aislamientos de $S$. pneumoniae.

\section{MATERIALES Y MÉTODOS}

Se analizaron 8993 aislamientos de S. pneumoniae recuperados entre $2000 \mathrm{y}$
2005 de niños menores de 6 años con infecciones invasoras. Estos aislamientos procedían de 10 países latinoamericanos (Argentina, Brasil, Chile, Colombia, Cuba, México, Paraguay, República Dominicana, Uruguay y Venezuela) participantes en la segunda etapa del Programa de Vigilancia de Neumonías y Meningitis Bacterianas, de SIREVA II, coordinado por la OPS $(9,10)$.

En el cuadro 1 se presenta el número de aislamientos estudiados por país $\mathrm{y}$ año. De acuerdo con el diagnóstico, 3277 $(36,4 \%)$ procedían de casos de neumonía, $3213(35,7 \%)$ de meningitis, $2155(24,0 \%)$ de sepsis o bacteriemia y $348(3,9 \%)$ de otras enfermedades invasoras.

\section{Sensibilidad antimicrobiana}

Se determinó la sensibilidad de los aislamientos a la eritromicina $(15 \mu \mathrm{g})$, el cloranfenicol $(30 \mu \mathrm{g})$, el SXT $(1,25 /$ $23,7 \mu \mathrm{g})$ y la vancomicina $(30 \mu \mathrm{g})$ por el método de difusión con discos de Kirby Bauer y la concentración inhibitoria mínima (CIM) por el método de microdilución en caldo, dilución en agar o la prueba denominada E-test. Las pruebas se realizaron de acuerdo con la metodología estandarizada en SIREVA II (12) y las recomendaciones e interpretación del Instituto de Estándares Clínicos y de Laboratorio (CLSI) (13-15). Como control se utilizó la cepa $S$. pneumoniae ATCC 49619.

CUADRO 1. Aislamientos de Streptococcus pneumoniae por país y año de vigilancia. SIREVA II, 2000-2005

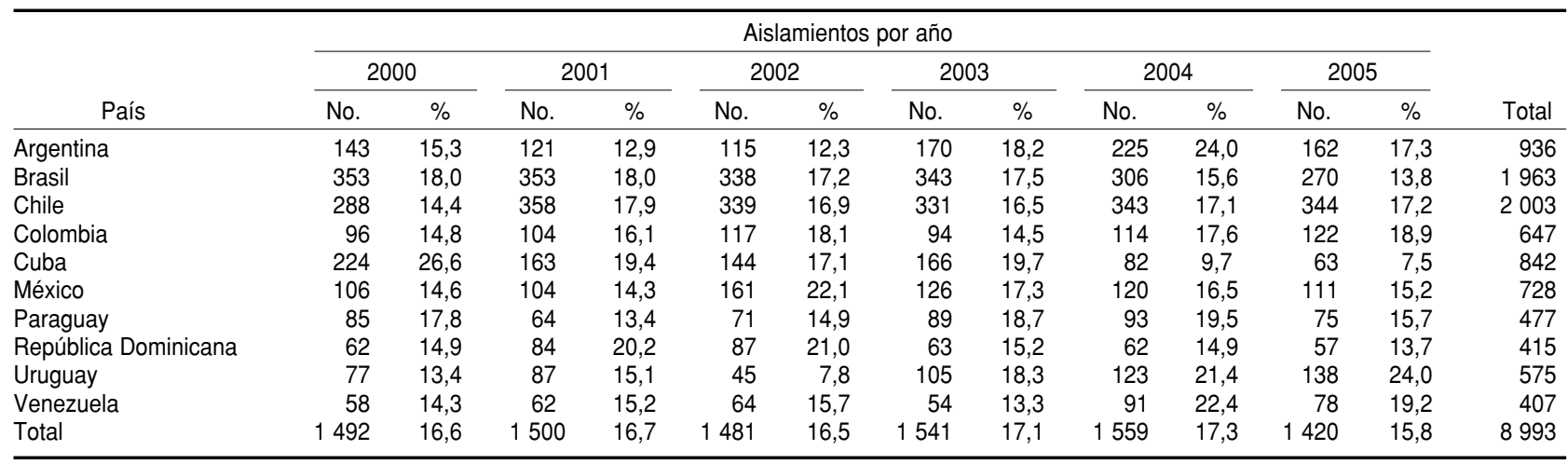


La resistencia a múltiples antibióticos o multirresistencia se definió como la resistencia a tres o más familias de antibióticos, ya fueran de los no betalactámicos analizados en este estudio o de los betalactámicos evaluados en un estudio previo (11).

\section{Análisis}

El análisis de los datos se realizó mediante el programa Epi-Info versión 6.04 (16). Para determinar la diferencia estadística entre las frecuencias se utilizó la prueba de la ji al cuadrado o la exacta de Fisher, según fuera el caso. Se determinó el riesgo relativo (RR) con sus intervalos de confianza de 95\% (IC95\%). El nivel de significación fue de $P<0,05$.

En los casos de Argentina, Chile, Colombia, México y Uruguay se comparó la frecuencia de aislamientos resistentes en el período 2000-2005 con el informado en 1994-1999 (8).

\section{Validación de los resultados}

El Programa Internacional de Evaluación Externa del Desempeño, coordinado por el Centro Nacional para el Streptococcus de Canadá, validó la calidad de los resultados de Brasil y Colombia, mientras que el Instituto Adolfo Lutz, de Brasil, validó los resultados de los laboratorios de Argentina, Chile, Cuba, Paraguay, República Dominicana, Uruguay y Venezuela, y el Instituto Nacional de Salud, de Colombia, validó los de México. El programa de validación abarcó las pruebas confirmatorias de identificación, serotipificación y de sensibilidad antimicrobiana y forma parte del sistema de gestión de la calidad de SIREVA II (17).

\section{RESULTADOS}

Todos los aislamientos analizados fueron sensibles a la vancomicina.

\section{Resistencia al SXT}

La sensibilidad al SXT se analizó en 8401 aislamientos $(93,4 \%$ de los 8993 aislamientos estudiados). La resistencia a este antibiótico fue la más frecuente en los 10 países analizados: $930(11,1 \%)$ aislamientos presentaron resistencia intermedia (mínimo: 4,6\%; máximo: 16,3\%) y $3808(45,3 \%)$ alta resistencia (mínimo: 15,5\%; máximo: $68,4 \%$ ), para un total de $4738(56,4 \%)$ aislamientos con algún nivel de resistencia. La mayor proporción de aislamientos resistentes se observó en Brasil $(71,9 \%)$ y la más baja en Chile $(43,1 \%)$ (cuadro 2).

Al comparar los datos de los años 2000 y 2005 se observó un incremento significativo en la proporción de aislamientos resistentes a este antibiótico en México (de $57,0 \%$ a $72,0 \%$; RR = 1,26; IC95\%: 1,01 a 1,$58 ; P=0,03$ ), Uruguay (de $43,4 \%$ a $87,1 \% ; \mathrm{RR}=1,55 ;$ IC95\%: 1,15 a 2,09 ; $P<0,01)$ y Venezuela (de $29,3 \%$ a $60,0 \%$; RR = 2,05; IC95\%: 1,32 a 3,18; $P<0,01$ ). En Cuba, se observó una drástica reducción entre las frecuencias detectadas en 2002 y 2005 (de 79,9\% a 23,8\%; RR = 0,54; IC95\%: 0,34 a 0,$85 ; P=0,002$ ) (figura $1 \mathrm{~A}$ ). Entre los períodos 1994-1999 y 20002005 se incrementó significativamente el promedio de la frecuencia de aislamientos resistentes en Colombia (de 35,0\% a $63,5 \%$; RR = 1,81; IC95\%: 1,58 a 2,04 ; $P<0,01$ ) y México (de 53,3\% a $69,4 \%$; $\mathrm{RR}=1,29 ; \mathrm{IC} 95 \%$ : 1,16 a 1,44; $P<0,01)$.

\section{Resistencia a la eritromicina}

De los 8544 aislamientos a los que se determinó la sensibilidad a la eritromicina (95,0\% del total), 108 (1,2\%) presentaron resistencia intermedia y 1211 $(14,2 \%)$ alta resistencia, para un total de $1319(15,4 \%)$ cepas con alguna resistencia a este antibiótico. Las mayores proporciones de aislamientos con resistencia procedían de México $(38,2 \%)$ y Venezuela $(32,9 \%)$, mientras que en Colombia, Paraguay y Brasil se observaron las frecuencias más bajas $(4,3 \%, 5,5 \%$ y $6,1 \%$, respectivamente) (cuadro 2 ).

CUADRO 2. Frecuencias absoluta y relativa (\%) de los aislamientos resistentes a tres antibióticos no betalactámicos, ${ }^{\text {a }}$ por país. SIREVA II, 2000-2005

\begin{tabular}{|c|c|c|c|c|c|c|c|}
\hline \multirow[b]{3}{*}{ País } & \multirow[b]{3}{*}{ Aislamientos } & \multicolumn{6}{|c|}{ Aislamientos resistentes $\mathrm{a}^{\mathrm{a}}$} \\
\hline & & \multicolumn{2}{|c|}{$\begin{array}{c}\mathrm{SXT}^{\mathrm{b}} \\
(n=8401)\end{array}$} & \multicolumn{2}{|c|}{$\begin{array}{c}\text { Eritromicina } \\
(n=8544)\end{array}$} & \multicolumn{2}{|c|}{$\begin{array}{c}\text { Cloranfenicol } \\
(n=8544)\end{array}$} \\
\hline & & No. & $\%$ & No. & $\%$ & No. & $\%$ \\
\hline Argentina & 936 & 488 & 52,1 & 99 & 10,6 & 9 & 1,0 \\
\hline Brasil & 1974 & 1419 & 71,9 & 120 & 6,1 & 11 & 0,6 \\
\hline Chile & 1700 & 732 & 43,1 & 475 & 27,9 & 35 & 2,1 \\
\hline Colombia & 647 & 411 & 63,5 & 28 & 4,3 & 32 & 4,9 \\
\hline Cuba & 842 & 418 & 49,6 & 104 & 12,4 & 145 & 17,2 \\
\hline México & 571 & 396 & 69,4 & 218 & 38,2 & 98 & 17,2 \\
\hline Paraguay & 477 & 244 & 51,2 & 26 & 5,5 & 14 & 2,9 \\
\hline República Dominicana & 415 & 145 & $53,3^{c}$ & 51 & 12,3 & 6 & 1,4 \\
\hline Uruguay & 575 & 299 & 52,0 & 64 & 11,1 & 6 & 1,0 \\
\hline Venezuela & 407 & 186 & 45,7 & 134 & 32,9 & 37 & 9,1 \\
\hline Total & 8544 & 4738 & $56,4^{\mathrm{d}}$ & 1319 & 15,4 & 393 & 4,6 \\
\hline
\end{tabular}

a Suma de resistencia intermedia y alta resistencia. Todos los aislamientos fueron sensibles a la vancomicina. b SXT: trimetoprim-sulfametozaxol.

cEn República Dominicana solo se evaluaron 272 aislamientos con este antibiótico.

d Calculado a partir de los 8401 aislamientos evaluados con este antibiótico.
Al comparar la frecuencia de los aislamientos resistentes a la eritromicina en el gura 1B), se observaron incrementos significativos en Argentina (de 6,3\% a 14,2\% Chile (de $6,3 \%$ a $15,9 \%$; RR = 2,56; IC $95 \%$ : $1,54$ a 4,$25 ; P<0,001)$ y Cuba (de $4,9 \%$ a $15,9 \% ; \mathrm{RR}=3,23$; IC95\%: 1,44 a 7,26 ; $P=0,003)$, y en la frecuencia promedio de todos los países (de $8,4 \%$ a $13,7 \%$; $\mathrm{RR}=1,64$; IC95\%: 1,32 a 2,03; $P<0,001$ ). Esta tendencia también se observó al parar los datos de los períodos 1994 1999 y 2000-2005 en Argentina (de 2,1\% a $10,6 \%$; RR = 4,96; IC95\%: 3,12 a 7,89; $P<0,01$ ), México (de 22,2\% a 38,2\% $\mathrm{R}=1,67 ; \mathrm{IC} 95 \%: 1,35$ a 2,$06 ; P<0,01) \mathrm{y}$ Uruguay (de $3,7 \%$ a $11,1 \%$; $R R=2,98$; IC95\%: 1,63 a 5,44; $P<0,01)$.

\section{Resistencia al cloranfenicol}

La sensibilidad al cloranfenicol se determinó en 8544 aislamientos, de los cuales $393(4,6 \%)$ presentaron alta resistencia. Cuba y México presentaron la mayor proporción de aislamientos resistentes $(17,2 \%)$ y Brasil la más baja $(0,6 \%)$ (cuadro 2). Cuando se compararon los del año 2000 y del 2005 se observó de biótico en Colombia (de $8,3 \%$ a $1,6 \%$; $\mathrm{RR}=0,20$; IC95\%: 0,04 a 0,91; $P=0,02) \mathrm{y}$ México (de 28,0\% a 11,0\%; $R R=0,39$; IC95\%: 0,20 a 0,79; $P<0,01$ ) (figura 1C); cuando se compararon los dos períodos de vigilancia (1994-1999 y 2000-2005) se encontró una disminución similar en Coaño 2000 con la encontrada en 2005 (fi- 
FIGURA 1. Aislamientos resistentes (\%) a tres antibióticos no betalactámicos, por país y año de vigilancia. SIREVA II, 2000 a 2005

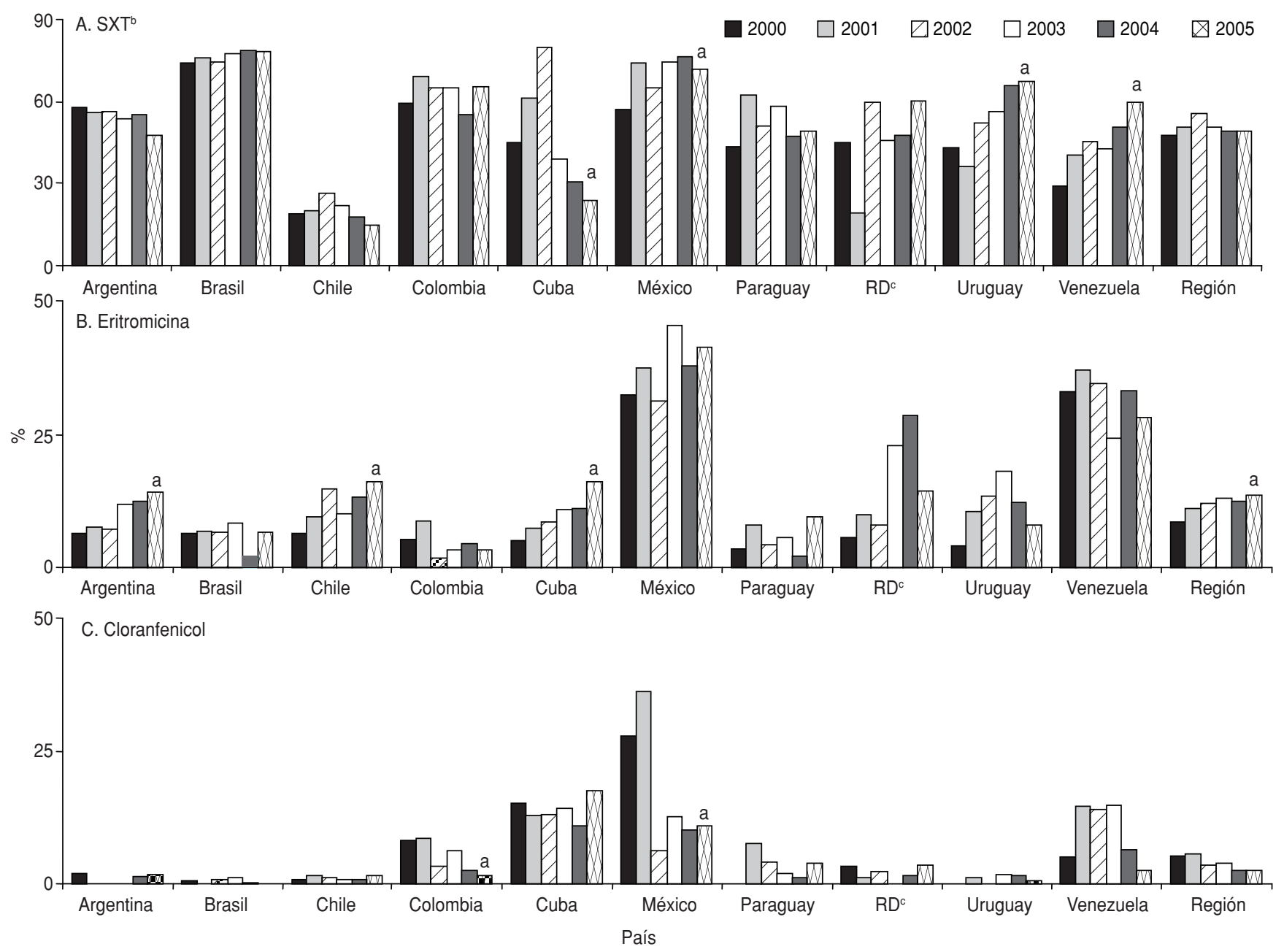

a Diferencia significativa entre las frecuencias de 2000 y $2005(P<0,05)$.

b SXT: trimetoprim sulfametoxazol.

${ }^{\mathrm{c}} \mathrm{RD}$ : República Dominicana.

lombia (de 12,6\% a 4,9\%; RR $=0,40$; IC95\%: 0,27 a 0,59; $P<0,01$ ) y México (de $52,9 \%$ a $17,2 \%$; RR $=0,31$; IC95\%: 0,25 a 0,38; $P<0,01)$.

\section{Resistencia por diagnóstico}

Se observó una frecuencia significativamente mayor de aislamientos con algún grado de resistencia al SXT en los aislamientos recuperados de neumonía $(61,6 \%)$ que en los casos de meningitis (56,5\%; RR = 1,09; IC95\%: 1,04 a 1,14; $P<0,01)$; mientras que para la eritromicina, la proporción de aislamientos resistentes fue mayor en los pacientes con sepsis $(25,5 \%)$ y otras enfermedades invasoras $(18,5 \%)$ que en los casos de neumonía (13,2\%; RR = 1,80; IC95\%: 1,60 a 2,$01 ; P<0,01)$ y meningitis $(11,1 \%$; $\mathrm{RR}=2,23$; IC95\%: 1,97 a 2,52; $P<0,01$ ).
No se observaron diferencias significativas en cuanto a la frecuencia de la resistencia al cloranfenicol en los aislamientos de las diferentes fuentes (cuadro 3).

\section{Resistencia según el serotipo de S. pneumoniae}

El análisis de la frecuencia de la resistencia a los antibióticos estudiados según el serotipo se presenta en el cuadro 4. Cuando se comparó la resistencia de cada uno de los serotipos con el promedio de resistencia a cada uno de los antibióticos, los serotipos 5, 6B, 9V, 14, $19 \mathrm{~F}$ y $23 \mathrm{~F}$ presentaron una mayor resistencia al SXT; la resistencia en los serotipos $6 \mathrm{~B}, 14$ y $19 \mathrm{~F}$ a la eritromicina fue mayor que el promedio, al igual que la resistencia en los serotipos $6 \mathrm{~B}$ y $23 \mathrm{~F}$ al cloranfenicol (cuadro 4).

\section{Multirresistencia}

La resistencia a múltiples antibióticos se determinó en 808 aislamientos $(9,5 \%$ de los 8469 que tenían el dato de susceptibilidad a los cinco antibióticos analizados). Las frecuencias más bajas se encontraron en Cuba $(0,7 \%)$ y Brasil $(1,3 \%)$, mientras las más altas se observaron en México $(48,5 \%)$ y Venezuela (25\%). El patrón más frecuente de multirresistencia fue a la penicilina, el SXT y la eritromicina, observado en $406(50,2 \%)$ de los aislamientos estudiados (cuadro 5).

\section{DISCUSIÓN}

El programa SIREVA ha mantenido la vigilancia de la resistencia bacteriana a los antibióticos durante 13 años en América Latina y el Caribe $(3-5,9-10)$ y es el 
CUADRO 3. Frecuencias absoluta y relativa (\%) de los aislamientos resistentes a tres antibióticos no betalactámicos, por diagnóstico clínico de la fuente de aislamiento y grado de resistencia. SIREVA II, 2000-2005

\begin{tabular}{|c|c|c|c|c|c|c|c|c|}
\hline \multirow[b]{3}{*}{ Antibiótico } & \multirow{3}{*}{$\begin{array}{c}\text { Diagnóstico } \\
\text { del caso } \\
\text { fuente }\end{array}$} & \multirow[b]{3}{*}{ Aislamientos } & \multicolumn{6}{|c|}{ Aislamientos según su resistencia ${ }^{a}$} \\
\hline & & & \multicolumn{2}{|c|}{ Sensibles } & \multicolumn{2}{|c|}{ Intermedia } & \multicolumn{2}{|c|}{ Alta } \\
\hline & & & No. & $\%$ & No. & $\%$ & No. & $\%$ \\
\hline \multirow[t]{4}{*}{$S T X^{b}$} & Neumonía & 3061 & 1176 & 38,4 & 328 & 10,7 & 1557 & $50,9^{c}$ \\
\hline & Meningitis & 3066 & 1333 & 43,5 & 355 & 11,6 & 1378 & 44,9 \\
\hline & Sepsis & 1876 & 1009 & 53,8 & 187 & 10,0 & 680 & 36,2 \\
\hline & Otros $^{d}$ & 398 & 145 & 36,4 & 60 & 15,1 & 193 & $48,5^{c}$ \\
\hline \multirow[t]{4}{*}{ Eritromicina } & Neumonía & 3172 & 2753 & 86,8 & 34 & 1,1 & 385 & 12,1 \\
\hline & Meningitis & 3109 & 2765 & 88,9 & 52 & 1,7 & 292 & 9,4 \\
\hline & Sepsis & 1955 & 1456 & 74,5 & 20 & 1,0 & 479 & $24,5^{c}$ \\
\hline & Otros $^{d}$ & 308 & 251 & 81,5 & 2 & 0,6 & 55 & $17,9^{c}$ \\
\hline \multirow[t]{4}{*}{ Cloranfenicol } & Neumonía & 3172 & 3036 & 95,7 & & & 136 & 4,3 \\
\hline & Meningitis & 3124 & 2955 & 94,6 & & & 169 & 5,4 \\
\hline & Sepsis & 1848 & 1780 & 96,3 & & & 68 & 3,7 \\
\hline & Otros $^{d}$ & 400 & 380 & 95,0 & & & 20 & 5,0 \\
\hline
\end{tabular}

a Según el método de difusión con discos de Kirby Bauer (12). Sensibles: halo de inhibición $\geq 19 \mathrm{~mm}$ para el SXT y $\geq 21 \mathrm{~mm}$ para la eritromicina y el cloranfenicol. Intermedia: halo de 16 a $18 \mathrm{~mm}$ para el SXT y de 16 a $20 \mathrm{~mm}$ para la eritromicina; no aplica para el cloranfenicol. Alta: halo $\leq 15 \mathrm{~mm}$ para el SXT y la eritromicina y $\leq 20 \mathrm{~mm}$ para el cloranfenicol.

b SXT: trimetoprim-sulfametozaxol.

${ }^{C}$ Diferencia significativa con respecto al total de resistencia entre las diferentes enfermedades según la prueba de la ji al cuadrado $(P<0,01)$.

¿ Otros: diagnósticos de otras enfermedades invasoras.

primer esfuerzo internacional para la vigilancia de laboratorio de los serotipos y la susceptibilidad antimicrobiana de $S$. pneumoniae en países en desarrollo. Esto hace de SIREVA un modelo colaborativo para la Región en este campo.

\section{Resistencia al SXT}

La resistencia de S. pneumoniae al SXT fue la más frecuente de los cuatro antibióticos estudiados y su incremento en los cinco países en los que se compararon los períodos 1994-1999 (8) y 20002005 fue significativo (de $45,6 \%$ a 50,6\%; $P<0,001)$, con los mayores incrementos en Colombia y México. Entre los años 2000 y 2005, solamente se observaron reducciones en la frecuencia de los aislamientos resistentes, aunque no significativas, en Chile - posiblemente como resultado del programa de control del uso de antibióticos que mantiene ese país desde 1999 (18) — y en Cuba, donde la frecuencia disminuyó drásticamente después del año 2002. Otros países también han informado altas frecuencias de aislamientos de esta bacteria resistentes al SXT, como Taiwán (87\%) (19), España $(66,5 \%)$, Francia $(42,0 \%)$ e Italia $(41,1 \%)$ (20). Según Klugman, la alta resistencia al SXT está asociada con la mayor resistencia a la penicilina y a la ceftriaxona (21), lo cual coincide con los resultados obtenidos por SIREVA II en el período
2000-2005: 37,8\% de los aislamientos estudiados era resistente a la penicilina $(22,1 \%$ con resistencia intermedia $y$ $15,7 \%$ con alta resistencia) y $14,5 \%$ a las cefalosporinas de tercera generación (11,4\% con resistencia intermedia y $3,1 \%$ con alta resistencia) (11).

\section{Resistencia a la eritromicina}

Al comparar los presentes resultados con los obtenidos por SIREVA en el período 1994-1999 (3) se observó un incremento significativo en la resistencia de $S$. pneumoniae a la eritromicina en Argentina, México y Uruguay $(P<0,001)$. En promedio, la frecuencia de aislamientos resistentes a la eritromicina en los 10 países estudiados $(15,4 \%)$ fue similar a la informada en Canadá en el año 2000 en aislamientos de infecciones invasoras y no invasoras en la población general (22). Se deben resaltar tres hallazgos de gran importancia: la mayor resistencia a este antibiótico observada en México y Venezuela, el incremento significativo en la resistencia en países como Argentina, Chile y Cuba entre los años 2000 y 2005, y el aumento constatado en la República Dominicana en los años 2003 y 2004 (figura 1B).

Klugman y Lonks informaron del aumento general de la resistencia a la eritromicina observado en el mundo (23) y lo relacionaron con el uso de este antibiótico en el tratamiento empírico de la in- fección respiratoria aguda adquirida en la comunidad. Dias y colaboradores, en un estudio realizado en Portugal en pacientes adultos, observaron un incremento en la resistencia a la eritromicina de $4,0 \%$ a $23,2 \%$ entre 1994 a 2000 , con un promedio anual de 19\% (24). En un estudio de vigilancia realizado en ocho países europeos entre 2001 a 2003 también se encontró un aumento en la frecuencia de los aislamientos resistentes a los macrólidos (claritromicina) obtenidos de adultos con infecciones invasoras y no invasoras; las mayores frecuencias se observaron en Francia $(46,1 \%)$ y España $(43,6 \%)$. En otro análisis realizado en España se encontró que $40,1 \%$ de los aislamientos obtenidos de niños menores de 5 años con infecciones invasoras eran resistentes a la eritromicina (25). Además, $55,6 \%$ de los aislamientos con susceptibilidad disminuida a la penicilina también resultaron resistentes a la eritromicina (25). Estas frecuencias son similares a las encontradas ahora en México $(38,2 \%)$ y Venezuela $(32,9 \%)$. Las menores frecuencias de aislamientos resistentes observadas en el estudio europeo correspondieron a Austria $(10,0 \%)$ y Portugal $(10,3 \%)$ (20), aún así, más altas que las informadas aquí para Colombia (4,3\%), Paraguay $(5,5 \%)$ y Brasil $(6,1 \%)$. Los autores atribuyen esta resistencia al uso de macrólidos en el tratamiento de enfermedades invasoras causadas por aislamientos de S. pneumoniae resistentes a la penicilina (20).

\section{Resistencia al cloranfenicol}

Al comparar estos resultados con los obtenidos por SIREVA entre 1994 y 1999 (3) se observó una disminución general en la resistencia (de $11,5 \%$ a $3,9 \%$; $P<0,001)$, especialmente en Colombia (de $12,6 \%$ a $4,9 \%$ ) y México (de $52,9 \%$ a $17,2 \%$ ). La frecuencia de aislamientos resistentes al cloranfenicol observada en la mayoría de los países participantes en SIREVA II resultó similar a la informada en Canadá $(2,2 \%)$ (22) y en algunos países europeos participantes en el Sistema Europeo de Vigilancia de las Resistencias a los Agentes Antimicrobianos (20), como Italia $(6,7 \%)$ (26), Bélgica $(2,7 \%)$ (20) y Portugal (2,3\%) (24). Por su parte, los valores más elevados, encontrados en México, Cuba y Venezuela, son similares a los de Francia $(14,7 \%)$ y España $(25,8 \%)$ (20). En Túnez (27) se encontró que $21 \%$ de los aislamientos resistentes a la eritromicina lo era también al cloranfenicol, 
CUADRO 4. Frecuencias absoluta y relativa (\%) de los aislamientos resistentes a tres antibióticos no betalactámicos, por serotipo y grado de resistencia. SIREVA II, 2000-2005

\begin{tabular}{|c|c|c|c|c|c|c|c|c|c|c|}
\hline \multirow[b]{3}{*}{ Antibiótico/serotipo } & \multirow[b]{3}{*}{ Aislamientos } & \multicolumn{6}{|c|}{ Aislamientos con resistencia ${ }^{a}$} & \multirow{3}{*}{$\begin{array}{l}\text { Riesgo } \\
\text { relativo }\end{array}$} & \multirow[b]{3}{*}{ IC $95 \%{ }^{b}$} & \multirow[b]{3}{*}{$P^{\mathrm{c}}$} \\
\hline & & \multicolumn{2}{|c|}{ Intermedia } & \multicolumn{2}{|c|}{ Alta } & \multicolumn{2}{|c|}{ Total } & & & \\
\hline & & No. & $\%$ & No. & $\%$ & No. & $\%$ & & & \\
\hline \multicolumn{11}{|l|}{$S X T^{d}$} \\
\hline 1 & 644 & 60 & 9,3 & 128 & 19,9 & 188 & 29,2 & $\mathrm{NR}^{\mathrm{e}}$ & NR & NR \\
\hline 3 & 176 & 3 & 1,7 & 7 & 4,0 & 10 & 5,7 & NR & NR & NR \\
\hline 4 & 131 & 11 & 8,4 & 12 & 9,2 & 23 & 17,6 & NR & NR & NR \\
\hline $7 \mathrm{~F}$ & 289 & 17 & 5,9 & 15 & 5,2 & 32 & 11,1 & NR & NR & NR \\
\hline $9 \mathrm{~V}$ & 244 & 14 & 5,7 & 164 & 67,2 & 178 & 73,0 & 1,32 & $1,22-1,42$ & $<0,01$ \\
\hline 14 & 2491 & 136 & 5,5 & 1837 & 73,7 & 1973 & 79,2 & 1,45 & $1,41-49$ & $<0,01$ \\
\hline $18 \mathrm{C}$ & 514 & 53 & 10,3 & 105 & 20,4 & 158 & 30,7 & NR & NR & NR \\
\hline $19 \mathrm{~A}$ & 317 & 31 & 9,8 & 113 & 35,6 & 144 & 45,4 & NR & NR & NR \\
\hline $19 \mathrm{~F}$ & 484 & 65 & 13,4 & 247 & 51,0 & 312 & 64,5 & 1,26 & $1,18-1,34$ & $<0,01$ \\
\hline $23 \mathrm{~F}$ & 391 & 60 & 15,3 & 189 & 48,3 & 249 & 63,7 & 1,17 & $1,08-1,26$ & $<0,01$ \\
\hline \multicolumn{11}{|l|}{ Eritromicina } \\
\hline 4 & 131 & 1 & 0,8 & 2 & 1,5 & 3 & 2,3 & NR & NR & NR \\
\hline 5 & 603 & 3 & 0,5 & 4 & 0,7 & 7 & 1,2 & NR & NR & NR \\
\hline $6 \mathrm{~A}$ & 311 & 5 & 1,6 & 49 & 15,8 & 54 & 17,4 & NR & NR & NR \\
\hline $6 \mathrm{~B}$ & 784 & 9 & 1,1 & 216 & 27,6 & 225 & 28,7 & 1,85 & $1,64-2,09$ & $<0,01$ \\
\hline $7 \mathrm{~F}$ & 289 & 9 & 3,1 & 5 & 1,7 & 14 & 4,8 & NR & NR & NR \\
\hline $9 \mathrm{~V}$ & 244 & 5 & 2,0 & 28 & 11,5 & 33 & 13,5 & NR & NR & NR \\
\hline 14 & 2491 & 18 & 0,7 & 636 & 25,5 & 654 & 26,3 & 1,70 & $1,56-1,84$ & $<0,01$ \\
\hline $18 \mathrm{C}$ & 514 & 10 & 1,9 & 11 & 2,1 & 21 & 4,1 & NR & NR & NR \\
\hline $19 \mathrm{~A}$ & 317 & 6 & 1,9 & 24 & 7,6 & 30 & 9,5 & NR & NR & NR \\
\hline $19 \mathrm{~F}$ & 484 & 16 & 3,3 & 110 & 22,7 & 126 & 26,0 & 1,63 & $1,39-1,92$ & $<0,01$ \\
\hline $23 \mathrm{~F}$ & 391 & 13 & 3,3 & 67 & 17,1 & 80 & 20,5 & NR & NR & NR \\
\hline$N T^{f}$ & 139 & 2 & 1,4 & 19 & 13,7 & 21 & 15,1 & NR & NR & NR \\
\hline Otros & 950 & 7 & 0,7 & 34 & 3,6 & 41 & 4,3 & NR & NR & NR \\
\hline \multicolumn{11}{|l|}{ Cloranfenicol } \\
\hline $18 \mathrm{C}$ & 514 & & & 21 & 4,1 & 21 & 4,1 & NR & NR & NR \\
\hline $19 \mathrm{~A}$ & 317 & & & 9 & 2,8 & 9 & 2,8 & NR & NR & NR \\
\hline $19 \mathrm{~F}$ & 484 & & & 46 & 9,5 & 46 & 9,5 & NR & NR & NR \\
\hline $23 \mathrm{~F}$ & 391 & & & 80 & 20,5 & 80 & 20,5 & 4,39 & $3,53-5,47$ & $<0,01$ \\
\hline $\mathrm{N} \mathrm{T}^{f}$ & 139 & & & 4 & 2,9 & 4 & 2,9 & NR & NR & NR \\
\hline Otros & 950 & & & 22 & 2,3 & 22 & 2,3 & NR & NR & NR \\
\hline
\end{tabular}

a Según el método de difusión con discos de Kirby Bauer (12). Resistencia intermedia: halo de 16 a 18 mm para el SXT y de 16 a 20 mm para la eritromicina; no aplica para el cloranfenicol. Alta resistencia: halo $\leq 15 \mathrm{~mm}$ para el SXT y la eritromicina y $\leq 20 \mathrm{~mm}$ para el cloranfenicol.

b IC95\%: intervalo de confianza de 95\%.

${ }^{c}$ Diferencia significativa con respecto al promedio según la prueba de la ji al cuadrado $(P<0,05)$.

d SXT: trimetoprim-sulfametozaxol.

e NR: no realizado. El cálculo de significación se realizó únicamente en aquellos serotipos que tenían una frecuencia mayor que el promedio.

${ }^{\dagger}$ NT: no tipificable.

algo similar a lo observado en México y Venezuela. El surgimiento de la resistencia de S. pneumoniae al cloranfenicol puede estar asociado con el uso de ese antibiótico en el tratamiento de la meningitis bacteriana $\mathrm{y}$, generalmente, se combina con la resistencia a otros antimicrobianos (21).

\section{Resistencia según el serotipo de S. pneumoniae}

En este estudio, la resistencia al SXT estuvo asociada especialmente con los serotipos 5, 6B, 9V, 14, 19F y 23F. En un estudio realizado en Bulgaria, esta asociación se encontró con los serotipos 23F,
19F y el serogrupo 9 (28). La asociación de determinados serotipos con la resistencia a la eritromicina en los 10 países estudiados aquí confirmó lo observado en Portugal, donde los aislamientos correspondientes a los serogrupos 6 y 19 y el serotipo 14 mostraron una mayor frecuencia de resistencia (24). 
CUADRO 5. Patrones de resistencia múltiple a antibióticos betalactámicos y no betalactámicos en los aislamientos estudiados. SIREVA II, 2000 a 2005

\begin{tabular}{lcc}
\hline Patrón de resistencia $^{a}$ & Aislamientos & $\%$ \\
\hline P, SXT, E, C & 91 & 11,3 \\
P, SXT, E & 406 & 50,2 \\
P, SXT, C & 183 & 22,6 \\
SXT, C, E & 128 & 15,8 \\
Total & 808 & 100,0 \\
\hline
\end{tabular}

a P: penicilina; SXT: trimetoprim sulfametoxazol; E: eritromicina; C: cloranfenicol.

\section{Multirresistencia}

Es importante resaltar que se observó un incremento significativo en la frecuencia de la resistencia a múltiples antibióticos en este estudio $(9,5 \%)$ con respecto a lo informado por Hortal y colaboradores (8) para el período 19941999 (7,5\%; RR = 1,28; IC95\%: 1,11 a 1,48; $P<0,01)$. El patrón de resistencia más frecuente fue a la penicilina, el SXT y la eritromicina, con o sin resistencia al cloranfenicol (61,5\% de los aislamientos con resistencia múltiple); esto coincide con lo informado por Dias y colaboradores para Portugal (47\%) (24).

$\mathrm{Al}$ analizar estos resultados se deben tener presente algunas limitaciones. $\mathrm{Al}$ basarse solamente en los datos enviados por los laboratorios no se contó con los denominadores poblacionales, lo que impidió medir la carga de la enfermedad ocasionada por S. pneumoniae en la población estudiada. Por otra parte, debido a que esta vigilancia se realiza a través de los laboratorios y en forma voluntaria, podría haber interrupciones en el envío continuo de aislamientos a los centros de referencia. Sin embargo, la exitosa experiencia acumulada por SIREVA y SIREVA II con esta cohesionada red de laboratorios de América Latina y el Caribe, la gran cantidad de aislamientos estudiados y la calidad de los procedimientos de control realizados (17) validan los resultados generados y confirman la utilidad de esta información para diseñar y poner en marcha acciones preventivas y tomar decisiones sobre el esquema terapéutico adecuado para el tratamiento de las enfermedades invasoras ocasionadas por S. pneumoniae.

Por tanto, se puede concluir que en los 10 países de América Latina y el Caribe estudiados se observó un aumento en la frecuencia de aislamientos de S. pneumoniae resistentes al SXT y la eritromicina, así como una disminución en la proporción de aislamientos resistentes al cloranfenicol. Todos los aislamientos fueron sensibles a la vancomicina. Estas tendencias mostraron diferencias entre los países estudiados.

El incremento en la frecuencia de aislamientos resistentes a antibióticos observado en la mayoría de los países y su tendencia general a aumentar demuestran la necesidad de que los países implementen las políticas recomendadas por la OMS (7). En primer lugar, se debe controlar el uso indiscriminado de los antibióticos y reconsiderar su empleo en el tratamiento empírico de las enfermedades invasoras ocasionadas por S. pneumoniae, lo que contribuiría a detener esa tendencia.

Se debe mantener y fortalecer este tipo de vigilancia, ya que permite registrar los cambios en los patrones de resistencia a patógenos de interés para la salud pública en los países de la Región. Esta información debe tomarse en cuenta para tomar decisiones terapéuticas más adecuadas, que se traducirán en el empleo prudente de los antimicrobianos.

\section{Afiliación de los autores}

${ }^{1}$ Instituto Nacional de Salud, Bogotá, Colombia; ${ }^{2}$ Instituto Nacional de Enfermedades Infecciosas Dr. Carlos G. Malbrán, Buenos Aires, Argentina; ${ }^{3}$ Instituto Adolfo Lutz, Sâo Paulo, Brasil;'; Fundação Oswaldo Cruz, Sâo Paulo, Brasil; ${ }^{5}$ Instituto de Salud Pública, Santiago, Chile, ${ }^{6}$ Instituto de Medicina Tropical Pedro Kourí, La Habana, Cuba; ${ }^{7}$ Instituto Nacional de Salud Pública, Cuernavaca, Morelos, México; ${ }^{8}$ Instituto de Diagnóstico y Referencia Epidemiológicos, México, D.F., México, ${ }^{9}$ Laboratorio Central de Salud Pública, Asunción, Paraguay; ${ }^{10}$ Hospital Infantil Dr. Robert Reid Cabral, Santo Domingo, República Dominicana; ${ }^{11}$ Servicio Nacional de Laboratorios de Salud Pública, Montevideo, Uruguay; ${ }^{12}$ Instituto Nacional de Higiene Rafael Rangel, Caracas, Venezuela; ${ }^{13}$ Organización Panamericana de la Salud, Washington, D.C., Estados Unidos de América; ${ }^{14}$ Grupo SIREVA II: Argentina: Sofía Fossati, Paula Gagetti, Marisa Rodríguez y Elsa Chávez, Instituto Nacional de Enfermedades Infecciosas Dr. Carlos G. Malbrán, Buenos Aires. Brasil: Maria Luiza L. S. Guerra y Samanta Cristine G. Almeida, Instituto Adolfo Lutz, Sâo Paulo; Chile: Ingrid Heitmann, Bianca Rojas y Julio García, Instituto de
Salud Pública, Santiago; Rosanna Lagos y Mabel Seoane, Centro para Vacunas en Desarrollo, Santiago. Colombia: Olga Marina Sanabria, María Victoria Ovalle y Jaime Moreno, Instituto Nacional de Salud; Fernando de la Hoz, Universidad Nacional, Bogotá; Desiree Pastor, OPS, Bogotá. Cuba: Miriam Pérez Monrás, Gilda Toraño Peraza, Kiomy Fuentes Gort y Alina Llops Hernández, Instituto de Medicina Tropical Pedro Kourí, La Habana; Andrés Sambrano, Ministerio de Salud Pública, La Habana. México: María Noemí Carnalla Barajas, María Elena Velázquez-Meza, Instituto Nacional de Salud Pública, Cuernavaca, Morelos; José Luis Arredondo-García, Instituto Nacional de Pediatría, México, D.F.; Fortino Solórzano-Santos, Hospital de Pediatría, Centro Médico Nacional Siglo XXI, México, D.F.; Domingo SánchezFrancia, Hospital del Niño Morelense, Cuernavaca, Morelos; Patricia Gabino Noriega, Susana Jiménez Moreno, Enrique Herrera González y Sugei Janette Gámez Contreras, Instituto de Diagnóstico y Referencia Epidemiológicos, México, D.F.; Marco Antonio González García, Dirección General de Epidemiología, México, D.F. Paraguay: Rossana Franco, Laboratorio Central de Salud Pública, Asunción; Gloria Gómez, Hospital Nacional de Itaugua, Asunción; Juana Ortellado, Hospital de Clínicas, Asunción; Myrian Leguizamón, Instituto de Previsión Social, Asunción; Beatriz Quiñónez, Hospital General Pediátrico, Asunción. República Dominicana: Chabela Peña, Hilma Coradín, Josefina Fernández y Mirna Novas, Hospital Infantil Dr. Robert Reid Cabral, Santo Domingo; Gilda Tolari, Hospital Plaza de la Salud, Santo Domingo. Uruguay: Gabriel Pérez Giffoni, María Albini y Viviana Félix, Servicio Nacional de Laboratorios de Salud Pública, Montevideo; María Hortal, Hospital Pereira Rossell, Montevideo; Coral Fernández, Hospital Escuela del Litoral, Montevideo. Venezuela: Damarys Sánchez, Instituto Nacional de Higiene Rafael Rangel, Caracas.

Agradecimientos. Este estudio recibió financiamiento de la OPS, Canadian International Development Agency y cada uno de los países participantes. Se agradece la colaboración de los miembros del Grupo SIREVA Vigía, integrado por: Argentina: Cecilia Vescina, Hospital de Niños Sor Maria Ludovica, La Plata, Buenos Aires; Martha Von Specht, Hos- 
pital Provincial de Pediatría, Posadas; Diana Gómez y Victoria Monzani, Instituto Nacional de Epidemiología J. H. Jara y Hospital Materno Infantil Don V. Tetamanti, Mar del Plata; Clara Mayoral, Hospital de Niños Dr. O. Alassia, Santa Fe; Andrea Badano, Hospital de Niños Dr. V. J Vilela, Rosario; Claudia Hernández, Hospital de Pediatría Dr. J. P. Garrahan, Capital Federal; Cristina Pérez, Hospital Provincial Castro Rendón, Neuquén; María José Rial, Hospital de Niños Dr. Pedro de Elizalde, Capital Federal; Lydia Carvajal, Hospital de Niños Santísima Trinidad, Córdoba; Adriana Procopio, Hospital de Niños Dr. Ricardo Gutiérrez, Capital Federal. Brasil: Lucia R. Ferraz y Regilma Oliveira, Coordenação Geral dos Laboratórios de Saúde Pública, Sâo Paulo; Márcia L. Carvalho y Fernando Barros, Secretaria de Vigilância em Saúde, Ministério da Saúde; Profissionais de Rede de Laboratórios Centrais de Saúde Pública (LACENs) y Rede de Laboratórios Regionais do Instituto Adolfo Lutz, São Paulo; Telma R. M. P. Carvalhanas, Centro de Vigilância Epidemiológica, São Paulo; Cristiana Nascimento Carvalho, Hospital Clínicas, Faculdade de Medicina Federal, Bahia; Paulo Augustos M. Camargos, Hospital Universitário, Universidade Federal, Minas Gerais; Orlando Cesar Mantese, Hospital das Clínicas, Faculdade de Medicina Federal, Uberlândia; Ana Lucia S. S. Andrade, Departamento de Saúde Coletiva, Universidade Federal, Goiás; Ruben Schindler Maggi, Instituto Materno-Infantil, Recife; Eitan N. Berezin, Santa Casa, São Paulo. Chile: Servicios de Salud de Aconcagua, Antofagasta, Araucanía Norte, Araucanía Sur, Arauco, Arica, Atacama, Aysen Bío-Bío, Concepción, Coquimbo, Iquique, Llanchipal, Magallanes, Maule, Metropolitano Cen- tral, Metropolitano Norte, Metropolitano Occidente, Metropolitano Oriente, Metropolitano Sur, Metropolitano SurOriente, Nuble, O'Higgins, Osorno, Talcahuano, Valdivia, Valparaíso-San Antonio y Viña del Mar-Quillota. Colombia: Sandra Núñez, Laboratorio de Salud Pública (LSP), Bogotá; María Eugenia Gómez e Hilda Álvarez, LSP, Antioquia; María Beatriz Olaya y Martha Garner, LSP, Valle; Vianney Portilla, LSP, Santander; Alberto de la Ossa, LSP, Caldas; Luz Stella Álzate, LSP, Risaralda; Gladys Mora, LSP Norte, Santander; Martha Uzeta, Clínica del Niño, Bogotá; Cuba: Gerardo Martínez, Antonio Valdés, Antonio Pérez, Félix Dickinson, María del Carmen Batle, Instituto de Medicina Tropical Pedro Kourí, La Habana; Miguel A. Galindo, Ministerio de Salud Pública, La Habana. México: Verónica Andrade Almarz, Hospital del Niño Morelense, Cuernavaca, Morelos; Rosa María Hinojosa Robles, Hospital Universitario, Monterrey, Nuevo León; Rosario Vázquez Larios, Instituto Nacional de Cardiología, México, D.F.; Adolfo Pérez Miravete, Hospital Infantil de México, México, D.F.; Rayo Morfín Otero, Hospital Civil, Guadalajara, Jalisco; Fray Antonio Alcalde y Andrés Flores Santos, Hospital General, San Luis Potosí; Juan Carlos Tinoco Fávila, Hospital General, Durango; Margarita Gutiérrez Ahuatzin, Hospital del Niño Poblano, Puebla; Juan Manuel Barajas Magallón, Hospital Infantil, Morelia, Michoacán; Manuel Ybarra, Centro Médico Nacional Adolfo Ruiz Cortínez, Veracruz; Maricela Garzón Chiapa, Laboratorio Estatal de Salud Pública, Aguascalientes; Sergio Jesús López Sierra, Laboratorio Estatal de Salud Pública, Guanajuato; Laura Contreras Mioni, Laboratorio Estatal de Salud Pública, Puebla; Carmen Garza
Gallegos, Laboratorio Estatal de Salud Pública, Monterrey, Nuevo León; Héctor López López, Laboratorio Estatal de Salud Pública, Chihuahua; Irma Villegas Esquivel, Laboratorio Estatal de Salud Pública, Zacatecas; Sandra M. Suárez Moreno, Hospital Infantil Eva Sámano López Mateos, Morelia, Michoacán; Virginia Martínez Trejo y Hugo Caballero Novara, Hospital Pediátrico La Villa, México, D.F.; Eduardo Velázquez Medina, Hospital Pediátrico de Iztapalapa, México, D.F. Paraguay: Mario Martínez, Mercedes Carrillo, Mercedes Álvarez, Julio César Manzur y Héctor F. Fariña, Laboratorio Central de Salud Pública, Asunción; Edelira Ayala, Instituto de Medicina Tropical, Asunción; Miryan Rivas, Hospital de Clínicas, Asunción; Grismilda Gómez, Centro Materno Infantil, Asunción; Alba María Ropero, OPS, Asunción; Juan Irala, Hospital Militar, Asunción. República Dominicana: Olivia Henríquez, Hospital Materno Infantil San Lorenzo de Los Mina, Santo Domingo; Florencia Méndez, Hospital Dr. Luis Eduardo Aybar, Santo Domingo; Elba Holguín y Genara Santana, Hospital Dr. Arturo Grullón, Santiago; Milagro Peña, Hospital Infantil Dr. Robert Reid Cabral, Santo Domingo. Uruguay: Gabriela Algorta, Hospital Pereira Rossell, Montevideo; Mónica Finozzi, Hospital Salto, Salto; Isabel González, Casa de Galicia, Montevideo; Luis Jorge, Hospital de Treinta y Tres, Treinta y Tres; Nora Milanese, Hospital de Maldonado, Maldonado; Laura Pivel, IMPASA, Montevideo. Venezuela: Sofía Toro, Sandra Hernández, Betty Tarazona y Ángel Flores, Instituto Nacional de Higiene Rafael Rangel, Caracas; Lizbeth Camacho y María Victoria Benítez, Ministerio de Salud, Caracas; Amando Martí, Hospital Universitario, Caracas.

\section{REFERENCIAS}

1. Musher DM. Infections caused by Streptococcus pneumoniae: clinical spectrum, pathogenesis, immunity, and treatment. Rev Infect Dis. 1992;14(4):801-7.

2. Appelbaum PC. Resistance among Streptococcus pneumoniae: implications for drug selection. Clin Infect Dis. 2002;34(12):1613-20.

3. Di Fabio JL, Castañeda E, Agudelo CA, De La Hoz F, Hortal MD, Camou T, et al. Evolution of Streptococcus pneumoniae serotypes and penicillin susceptibility in Latin American, SIREVA-Vigía Group, 1993 to 1999. Pediatr Infect Dis J. 2001;20(10):959-67.
4. Agudelo CI, Moreno J, Sanabria O, Castañeda E. Streptococcus pneumoniae: evolución de los serotipos y los patrones de sensibilidad antimicrobiana en aislamientos invasivos en 11 años de vigilancia en Colombia (1994-2004). Grupo colombiano de trabajo en S. pneumoniae. Biomédica. 2006;26(2):234-49.

5. Brandileone MCC, Tadeu Casagrande $S$, Guerra MLLS, Zanella R, Andrade ALSS, Di Fabio JL. Increase in numbers of beta-lactamresistant invasive Streptococcus pneumoniae in Brazil and the impact of conjugate vaccine coverage. J Med Microbiol. 2006;55(5):567-74.
6. Faccone D, Andres P, Galas M, Tokumoto M, Rosato A, Corso A. Emergence of a Streptococcus pneumoniae clinical isolate highly resistant to telithromycin and fluoroquinolones. J Clin Microbiol. 2005;43(11):5800-3.

7. Organización Mundial de la Salud. Estrategia mundial de la OMS para contener la resistencia a los antimicrobianos. Ginebra: OMS; 2001. (WHO/CDS/CSR/DRS/2001.2.)

8. Hortal M, Lovgren M, De la Hoz F, Agudelo CI, Brandileone MC, Camou T, et al. Antibiotic resistance in Streptococcus pneumoniae in six Latin American countries: biological and 
therapeutic implications. Microbial Drug Resistance. 2001;7(4):391-401.

9. Gabastou JM, Agudelo CI, Brandileone MCC, Castañeda E, Lemos APS, Di Fabio JL, et al. Caracterización de aislamientos invasivos de S. pneumoniae, $H$. influenzae y N. meningitidis en América Latina y el Caribe: SIREVA II, 2000-2005. Rev Panam Salud Publica. 2008; 24(1):1-15.

10. Di Fabio JL, Homma A, Quadros C. Pan American Health Organization epidemiologic surveillance network for Streptococcus pneumoniae. Microb Drug Resist. 1997;3(2):131-3.

11. Castañeda E, Agudelo CI, Regueira M, Corso A, Brandileone MCC, Casagrande ST, et al. The SIREVA II project, 2000-2005: a laboratory based surveillance of Streptococcus pneumoniae invasive disease in children in ten Latin American countries. Ped Infect Dis J. (Aceptado para publicación).

12. Organización Panamericana de la Salud, Instituto Nacional de Salud de Colombia. Programa de vigilancia de los serotipos y resistencia antimicrobiana de Streptococcus pneumoniae y Haemophilus influenzae. Manual de procedimientos. Santa Fe de Bogotá: INS; 2004. Disponible en http://www.paho.org/ Spanish/AD/THS/EV/LABS-manual-vigi lancia-serotipos.pdf. Acceso el 1 de marzo de 2009.

13. Clinical and Laboratory Standards Institute. Performance standards for antimicrobial disk susceptibility test. 9th ed. Wayne, PA.: CLSI; 2006. (Document M2-A9. CLSI.)

14. Clinical and Laboratory Standards Institute. Methods for dilution antimicrobial susceptibility tests for bacteria that grow aerobically. 7th ed. Wayne, PA.: CLSI; 2006. (Document M7-A7. CLSI.)
15. Clinical and Laboratory Standards Institute. Performance standards for antimicrobial susceptibility testing. Wayne, PA.: CLSI; 2006. (Document M100-S16.)

16. Dean AG, Dean JA, Columbier D, Burton AH, Brendel KA, Smith DC, et al. Epi Info v. 6.0: a word processing, data-base and statistics program for epidemiology on microcomputers. Atlanta, GA: Centers for Disease Control and Prevention; 1994.

17. Lovgren M, Talbot JA, Brandileone MCC, Casagrande ST, Agudelo CI, Castañeda E, et al. Evolution of an international external quality assurance model on Streptococcus pneumoniae in Latin America: the SIREVA Project, 1993-2005. J Clin Microb. 2007;45(10):3184-90.

18. Bavestrello L, Cabello A, Casanova D. Impact of regulatory measures in the trends of community consumption of antibiotics in Chile. Rev Med Chil. 2002;130(11):1265-72.

19. Hsueh PR, Teng LJ, Lee LN, Yang PC, Ho SW, Luh KT. Extremely high incidence of macrolide and trimethoprim-sulfamethoxazole resistance among clinical isolates of Streptococcus pneumoniae in Taiwan. J Clin Microbiol. 1999;37(4):897-901.

20. Reinert RR, Reinert S, Van der Linden M, Cil MY, Al-Lahham A, Appelbaum P. Antimicrobial susceptibility of Streptococcus pneumoniae in eight European countries from 2001 to 2003. Antimicrob Agents Chemother. 2005; 49(7):2903-13.

21. Klugman KP. Pneumococcal resistance to antibiotics. Clin Microbiol Rev. 1990;3(2):171-96.

22. Low DE, De Azavedo J, Weiss K, Mazzulli T, Kuhn M, Church D, et al. Antimicrobial resistance among clinical isolates of Streptococcus pneumoniae in Canada during 2000. Antimicrob Agents Chemother. 2002;46(5):1295-301.
23. Klugman KP, Lonks JR. Hidden epidemic to macrolide-resistant pneumococci. Emerg Infect Dis. 2005;11(6):802-7.

24. Dias R, Louro D, Canica M. Antimicrobial susceptibility of invasive Streptococcus pneumoniae isolates in Portugal over an 11-year period. Antimicrob Agents Chemother. 2006; 50(6):2098-105.

25. Oteo J, Lazaro E, de Abajo FJ, Baquero F, Campos J, Spanish Members of the European Antimicrobial Resistance Surveillance System. Trends in antimicrobial resistance in 1,968 invasive Streptococcus pneumoniae strains isolated in Spanish hospitals (2001 to 2003): decreasing penicillin resistance in children's isolates. J Clin Microbiol. 2004;42(12): 5571-7.

26. Montagnani F, Stolzuoli L, Zanchi A, Cresti S, Cellesi C. Antimicrobial susceptibility of Streptococcus pyogenes and Streptococcus pneumoniae: surveillance from 1993 to 2004 in Central Italy. J Chemother. 2006;18(4):389-93.

27. Rachdi M, Boutiba-Ben Boubaker I, Moalla S, Smaoui H, Hammami A, Kechrid A, et al. Phenotypic and genotypic characterization of macrolide resistant Streptococcus pneumoniae in Tunisia. Pathol Biol. 2008;56(3):125-9.

28. Setchanova L, Tomasz A. Molecular characterization of penicillin-resistant Streptococcus pneumoniae isolates from Bulgaria. J Clin Microbiol. 1999;37(3):638-48.

Manuscrito recibido el 6 de abril de 2008. Aceptado para publicación, tras revisión, el 12 de octubre de 2008.

ABSTRACT Objective. To examine the development of resistance to erythromycin, chloramphenicol, trimethoprim-sulfamethoxazole (TMP-SMZ), and vancomycin of the invasive isolates of Streptococcus pneumoniae obtained from children in 10 Latin American/Caribbean countries during six years of surveillance.

Resistance to non-beta-lactam
antibiotics in the clinical
isolates of Streptococcus
pneumoniae of children in
Latin America. SIREVA II,
$2000-2005$ Methods. Analysis of 8993 isolates of S. pneumoniae recovered in 2000-2005 from children with invasive infections, who were less than 6 years of age, and from Argentina, Brazil, Chile, Colombia, Cuba, Dominican Republic, Mexico, Paraguay, Uruguay, or Venezuela. Antibiotic susceptibility was determined through the methods established and standardized by the SIREVA project. Multidrug resistance was defined as: resistance to three or more antibiotics of the same class; to the non-beta-lactams analyzed by this study; or, to the beta-lactams evaluated by a previous study, in which $37.8 \%$ of these isolates showed decreased susceptibility to penicillin.

Results. Some degree of resistance was found to TMP-SMZ and erythromycin (56.4\% and $15.4 \%$ of the isolates studied, respectively), with $4.6 \%$ highly resistant to chloramphenicol. All isolates were susceptible to vancomycin. The highest prevalence of TMP-SMZ resistance was observed in the pneumonia isolates; and that of erythromycin, in cases of sepsis $(61.6 \%$ and $25.5 \%$, respectively; $P<0.01)$. The highest prevalence of TMP-SMZ resistance was found in Brazil $(71.9 \%)$, and that of erythromycin, in Mexico (38.2\%) and Venezuela (32.9\%). The 14,6B, 19F, and 23F serotypes were most often associated with resistance to the antibiotics in the study. Conclusions. High and increasing rates of isolates resistant to TMP-SMZ and erythromycin were observed, as well as a decreasing percentage of isolates resistant to chloramphenicol. These trends highlight differences among the countries studied.

Key words Anti-bacterial agents, Streptococcus pneumoniae, beta-lactam resistance, child, Latin America, Caribbean. 